\title{
Classical eddy current losses in Soft Magnetic Composites
}

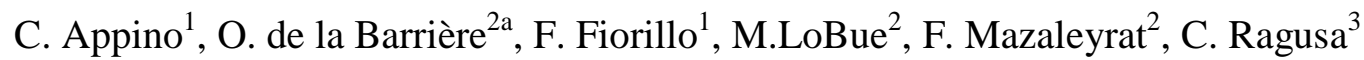

${ }^{1}$ Instituto Nazionale di Ricerca Metrologica (INRIM), Torino, Italy

${ }^{2}$ SATIE, ENS Cachan, CNRS, UniverSud, 61 av du President Wilson, F-94230 Cachan, France

${ }^{3}$ Dipartimento Energia, Politecnico di Torino, C.so Duca degli Abruzzi 24, 10129 Torino, Italy

$1 \quad$ Abstract

${ }^{\text {a }}$ Corresponding author: barriere@ satie.ens-cachan.fr 
This paper deals with the problem of loss evaluation in Soft Magnetic Composites (SMC), focusing

3 on the classical loss component. It is known that eddy currents can flow in these granular materials at

4 two different scales, that of the single particle (microscopic eddy currents) and that of the specimen

5 cross-section (macroscopic eddy currents), the latter ensuing from imperfect insulation between

6 particles. It is often argued that this macroscopic loss component can be calculated considering an

7 equivalent homogeneous material of same bulk resistivity. This assumption has not found so far clear

8 and general experimental validation. In this paper, we discuss energy loss experiments in two different

9 SMC materials, obtained using different binder types, and we verify that a classical macroscopic loss

10 component, the sole size-dependent term, can be separately identified. It is also put in evidence that,

11 depending on the material, the measured sample resistivity and the equivalent resistivity entering the

12 calculation of the macroscopic eddy currents may not be the same. A corrective coefficient is therefore

13 introduced and experimentally identified. This coefficient appears to depend on the material type only,

14 the role of sample shape and/or cross-sectional area being irrelevant. An efficient way to calculate the

15 macroscopic classical loss in these materials, based on a minimum set of preliminary experimental

16 results, is thus provided. In this way, a reliable procedure for loss separation, whatever the sample size,

17 can be implemented. 
20 Soft Magnetic Composites (SMC) are of interest in modern electrical engineering applications.

21 Their isotropic magnetic and thermal behavior provides a clear advantage for machines with 3D flux 22 paths, like axial flux machines [1][2], or claw pole generators [3].

23 The loss separation concept, associated with the Statistical Theory of Losses (STL) [4], is known to 24 efficiently assess the loss phenomenology in soft magnetic laminations. Its extension to SMC is, 25 however, far from simple, because one has to deal with an inhomogeneous granular structure, where 26 eddy currents (e.c.) flow at two different scales: the scale of the single particle (microscopic e.c.), and 27 the scale of the whole sample (macroscopic e.c., due to intergrain conductivity) [6][7][8]. The 28 correspondingly measured bulk resistivity is often considered in the macroscopic eddy current 29 calculations, assuming an equivalent homogeneous material. It has been suggested that microscopic 30 and macroscopic e.c. can be associated with microscopic and macroscopic classical loss components, 31 respectively [6]. Although a certain dependence of the total dynamic loss on the bulk resistivity has 32 been shown [9], no clear experimental evidence of the separation between macroscopic and 33 microscopic classical losses has been provided so far. At the same time, the assumed link between the 34 measured material resistivity and the macroscopic e.c. has not been supported by experimental 35 observations.

36 It was previously shown [10] that loss separation according to STL could be carried out in small and 37 highly resistive SMC samples, where the macroscopic eddy currents are negligible. This appears, 38 however, a substantial restriction when looking at a reliable loss prediction in electrical equipments 39 using SMC [11]. In this paper, this limitation is overcome, by considering different SMC samples of 40 various sizes using either organic or inorganic binders, with resistivity values spanning several orders 41 of magnitude. We start by putting in evidence the dependence of the specific dynamic loss on the 42 sample cross-sectional area. The loss component dependent on the sample size is singled out and found 43 to linearly depend on frequency, thereby justifying its assimilation to a classical loss. The problem of 
44 the relation between this macroscopic classical loss and the measured sample resistivity is discussed,

45 introducing a coefficient in the loss formulae that takes into account the grain-to-grain eddy current

46 percolation across random contacts. This theoretical framework is validated showing that the corrective

47 coefficient exclusively depends on the material type, regardless of the sample size. This provides an

48 efficient tool to make full loss decomposition in SMC, as discussed in the last part of the paper.

\section{I. EXPERIMENTAL}

\section{A. Samples}

51 The experiments presented in this paper have been carried out on several samples of two SMC materials, herein called $S M C_{1}$ and $S M C_{2}$, produced from a high purity iron powder ATOMET 1001HP [12] provided by Quebec Metal Powders (QMP). The particles in the $S M C_{1}$ and $S M C_{2}$ materials are

54 insulated by means of organic and inorganic binder, respectively. The $S M C_{1}$ material is heat-treated at low temperature $\left(1\right.$ hour at $\left.160^{\circ} \mathrm{C}\right)$, so as to improve the mechanical properties (e.g. fracture strength) without damaging the organic insulator [13]. A higher-temperature treatment (1 hour at $\left.425^{\circ} \mathrm{C}\right)$, as

57 permitted by the inorganic insulator, is applied to the $S M C_{2}$ material, bringing about a slight reduction 58 of the hysteresis (DC) loss contribution [13].

59 The samples are delivered as rings with rectangular cross-section (outside diameter $52.6 \mathrm{~mm}$, inside 60 diameter $43.8 \mathrm{~mm}$ ). Three different ring thicknesses have been considered: $t_{1}=5 \mathrm{~mm}, t_{2}=9 \mathrm{~mm}, t_{3}=$ $6113 \mathrm{~mm}$. Type and geometry of each sample are here identified as $S M C_{\mathrm{i}}-t_{\mathrm{j}}(\mathrm{i}=1,2$, and $\mathrm{j}=1,2,3)$. The compaction pressure was in all cases $p=600 \mathrm{MPa}$, resulting, however, in increased material density with decreasing sample thickness, as summarized in Table 1.

\section{B. Resistivity measurements}

In order to overcome the difficulties and ambiguities associated with the conventional four-point resistivity measurement [8][14], an indirect method, where the toroidal sample is used as the secondary 
68 resistivity of $S M C_{2}$ (inorganic binder) is more than one order of magnitude smaller than the one of

$69 S M C_{1}$ (organic binder). It is noted that different samples of a given material do not exactly exhibit the very same resistivity, because the manufacturing process is not perfectly reproducible.

\section{MODELING THE MACROSCOPIC EDDY CURRENT LOSSES}

\section{A. Macroscopic eddy current losses}

The magnetic characterization of the ring specimens is performed under controlled sinusoidal polarization (peak value $J_{\mathrm{p}}=1 \mathrm{~T}$ ) from DC to $10 \mathrm{kHz}$ with a calibrated hysteresisgraph-wattmeter, as described in [16]. The experiments show (see Fig. 1 for $S M C_{2}$, a similar behavior being observed in $S M C_{1}$ ) that the specific loss in SMC materials depends on the material cross-sectional area (i.e., ring thickness). To explain this phenomenon, it is often assumed [6][7] that the observed losses in SMC samples are due to physical effects occurring upon two different scales: a) the microscopic loss, due to the e.c. circulating within the individual iron particles; b) the macroscopic classical loss, due to the e.c. flowing from particle to particle thanks to imperfections in particle insulation and describing macroscopic patterns. However, no clear experimental evidence for effective role of these eddy currents has been provided so far and there is no consensus on the underlying assumptions [17][18]. In the following, we will provide evidence for a loss contribution depending on the sample cross-sectional area that appears to proportionally depend on frequency, as expected for a classical loss component.

In order to single out the contribution to the specific loss depending on the sample cross-section ( $W_{\text {MAC }}$ ) from the one occurring upon the scale of the single particle (the microscopic loss $W_{\text {MIC }}$ ), the loss difference measured in samples differing only for their size is considered. We thus write, considering two sizes (a) and (b): $\Delta W^{(\mathrm{a}, \mathrm{b})}=W^{(\mathrm{a})}-W^{(\mathrm{b})}=W_{\mathrm{MAC}}{ }^{(\mathrm{a})}-W_{\mathrm{MAC}}{ }^{(\mathrm{b})}$. Fig. 2 and Fig. 3 show the differences $\triangle W$ measured between $S M C_{1}-t_{3}$ and $S M C_{1}-t_{1}$, and $S M C_{2}-t_{3}$ and $S M C_{2}-t_{1}$, respectively. Similar results are obtained in other samples. $\Delta W$ linearly depends on frequency, thereby showing that the macroscopic loss contribution is classical in nature. 


$$
\Delta W^{(a, b)}=W^{(a)}-W^{(b)}=W_{\text {class, } \mathrm{MAC}}{ }^{(a)}-W_{\text {class, MAC }}{ }^{(b)} .
$$

92 We can thus generally write for the total specific loss $W\left(J_{\mathrm{p}}, f\right)=W_{\text {class,MAC }}\left(J_{\mathrm{p}}, f\right)+W_{\mathrm{MIC}}\left(J_{\mathrm{p}}, f\right)$. The

93 microscopic part $W_{\text {MIC }}$ was previously analyzed in the framework of STL [10] and was shown to be

94 equal to the sum of an hysteresis contribution, an excess component, and a classical loss term deriving

95 from the eddy currents circulating within the particles. This is defined as the microscopic classical loss

$96 W_{\text {class,MIC }}$.

\section{B. Link between the macroscopic classical loss component and the sample resistivity}

98 It is frequently assumed that the measured material resistivity can be directly used for the macroscopic

99 loss computation, assuming an equivalent homogeneous material [6][7]. But the link between sample 100 resistivity and macroscopic classical loss is not obvious, because, as shown in [19], percolation due to 101 random contacts between particles plays a role in highly compacted samples and interpretation of the 102 experiments calls for a specific model of conduction by random contacts [19]. But this model requires 103 considerable computational workload and a simpler approach is proposed here by introducing the 104 notion of equivalent resistivity for the loss $\rho^{\text {(loss) }}$, i.e. the resistivity which would produce, in an

105 homogeneous sample, the same macroscopic loss observed in the SMC. Due to percolation, $\rho^{(\text {loss })}$ is 106 expectedly different from the measured resistivity $\rho$, but we assume that proportionality exists, so that 107 we can write $\rho^{\text {(loss) }}=Q^{\text {(loss) }} \rho$, with $Q^{\text {(loss) }}$ a phenomenological coefficient. It is verified that $Q^{\text {(loss) }}$ 108 depends only on the type of material and can be obtained comparing two samples with different cross109 sectional area. Starting in fact from the calculation of eddy currents in a rectangular domain [10], we 110 consider a ring sample with rectangular cross-section (thickness $t$, width $\Delta R$, cross-sectional area $S_{\mathrm{c}}=$ $111 t \cdot \Delta R$ ) and we obtain the macroscopic classical loss as:

$$
W_{\text {class,MAC }}\left(J_{\mathrm{p}}, f\right)=2 \pi^{2} \frac{1}{\delta} \frac{1}{Q^{(\text {loss })} \rho} K_{\text {shape }}\left(\frac{\Delta R}{t}\right) \cdot S_{c} \cdot J_{\mathrm{p}}^{2} f
$$

112 where the parameter $K_{\text {shape, }}$ which depends only on the width-to-thickness ratio $\Delta R / t$, is computed 113 using a finite element method (it can be shown that the skin effect at the scale of the single particle is 
114 negligible, implying that $K_{\text {shape }}$ is independent of frequency). Comparing two samples (a) and (b) of the 115 same material, Eq. (2) can be written as:

$$
\Delta W^{(a, b)}=W^{(a)}-W^{(b)}=2 \pi^{2} \frac{1}{Q^{(\text {loss })}}\left\{\frac{K_{\text {shape }}^{(a)} S_{c}^{(a)}}{\delta^{(a)} \rho^{(a)}}-\frac{K_{\text {shape }}^{(b)} S_{c}^{(b)}}{\delta^{(b)} \rho^{(b)}}\right\} J_{\mathrm{p}}^{2} f \quad \quad[\mathrm{~J} / \mathrm{kg}]
$$

\section{7 \\ C. Validation of the macroscopic loss model}

118 In order to validate the macroscopic loss model, we show that the dimensionless coefficient $Q^{\text {(loss) }}$ is 119 independent of sample shape and size and is only material dependent. $Q^{(\text {loss })}$ is identified looking at the 120 experimentally observed loss difference between $S M C_{\mathrm{i}}-t_{2}(\mathrm{i}=1$ or 2$)$ and $S M C_{\mathrm{i}}-t_{1}$ samples. In fact, 121 since the experimental loss difference linearly depends on $f$, it is sufficient to adapt the coefficient $122 Q^{(\text {loss })}$ to get the observed behavior of $\Delta W^{(\mathrm{a}, \mathrm{b})}$ versus $f$. We find $Q^{(\text {loss })}=1$ for the material $S M C_{1}$ and $123 Q^{\text {(loss) }}=1.56$ for $S M C_{2}$. Since $Q^{(\text {loss })}=1$, the conventional approach invoking an equivalent 124 homogeneous material [6][7] is acceptable in calculating $W_{\text {class,MAC }}\left(J_{\mathrm{p}} f\right)$ in the material $\operatorname{SMC}_{1}$. This 125 implies that in the material with organic binder heat-treated at low temperature, eddy current 126 percolation by intergrain random contacts does not play any role (the observed resistivity being that of 127 the binder). On the other hand, in the material $S M C_{2}$, heat-treated at higher temperature, percolation 128 takes place and it is accordingly found that $\rho^{(\text {loss })}$ is higher than the measured resistivity [19]. This 129 points to percolation as a mechanism affecting to different extent the current patterns involved with the 130 conductivity measurements and the magnetic losses.

131 That the coefficient $Q^{(\text {loss })}$ is, to good approximation, material dependent only can be understood in 132 terms of local character of the random interparticle contacts, making $Q^{\text {(loss) }}$ independent of the cross133 sectional area in sufficiently big samples. This is an important point in the practical use of this model. 134 We observe in Fig. 2 and Fig. 3 the close behaviors of the experimental and the so calculated loss 135 differences $\Delta W$ versus $f$ in the ring samples $S M C_{\mathrm{i}}-t_{3}(\mathrm{i}=1$ or 2$)$ and $S M C_{\mathrm{i}}-t_{1}$ (the experimental $\Delta W$ observed at $f=0$ being related to the uncertainty associated with the determination of this quantity for 
137 the hysteresis loss component). The coefficient $Q^{\text {(loss) }}$ can then be simply obtained, for a given material, 138 from the loss difference measured on two differently sized samples, an important result in view of loss 139 prediction in practical cores.

\section{D. Loss separation}

141 Once the macroscopic classical loss is known, it is possible to perform the loss decomposition. The 142 microscopic classical loss (i.e. the classical loss at the scale of the single particle $W_{\text {class,MIC }}$ ) is calculated 143 once the size distribution of the particles is obtained by micrographic inspection [10]. In the present 144 experiments, $W_{\text {class,MIC }}$ is the same in $S M C_{1}$ and $S M C_{2}$ (the same iron powder is employed). The excess 145 and hysteresis loss components can then be singled out from the total experimental loss $W_{\text {tot }}$ (see [10] 146 for the detailed procedure). Fig. 4 and Fig. 5 present the results for $S M C_{1}-t_{2}$ and $S M C_{2}-t_{2}$, respectively 147 (sinusoidal polarization, $J_{\mathrm{p}}=1 \mathrm{~T}$ ). A striking difference in the macroscopic loss $W_{\text {class,MAC }}\left(J_{\mathrm{p},} f\right)$ between 148 the two materials is found, descending from the large difference in the measured resistivities (see Table 149 1). We note, in particular, that $W_{\text {class,MAC }}\left(J_{\mathrm{p}}, f\right) \sim 15 \cdot W_{\text {class,MIC }}\left(J_{\mathrm{p}}, f\right)$ in the $S M C_{2}-t_{2}$ sample. This would 150 restrict the use of $S M C_{2}$ to low frequencies.

\section{III. CONCLUSION}

152 We have put in evidence the link between sample resistivity and macroscopic classical loss in two 153 different classes of commercial Soft Magnetic Composites. An equivalent resistivity for the magnetic 154 losses, taking into account the effect of random interparticle contacts and percolation, has been 155 introduced besides the measured resistivity. It is a material related quantity, independent of the sample 156 size, which provides a simplified route to loss calculation in practical magnetic cores. 
[1] F. Marignetti, V.D. Colli, and S. Carbone, "Comparison of axial flux PM synchronous machines with different rotor back cores," IEEE Transactions on Magnetics, vol. 46, no. 2, pp. 598-601, 2010.

[2] H. Vansompel, P. Sergeant, and L. Dupré, "Optimized design considering the mass influence of an axial flux permanent-magnet synchronous generator with concentrated pole windings," IEEE Transactions on Magnetics, vol. 46, no. 12, pp. 4101-4107, 2010.

[3] L. Li, A. Kedous-Lebouc, A. Foggia, and J.C. Mipo, "Influence of magnetic materials on claw pole machines behavior," IEEE Transactions on Magnetics, vol. 46, no. 2, pp. 574-577, 2010.

[4] G. Bertotti, "General properties of power losses in soft ferromagnetic materials," IEEE Transactions on Magnetics, vol. 24, no. 1, pp. 621-630, 1988.

[5] E. Barbisio, F. Fiorillo, and C. Ragusa, "Predicting Loss in Magnetic Steels Under Arbitrary Induction Waveform and With Minor Hysteresis Loops," IEEE Transactions on Magnetics, vol. 40, no. 4, pp. 1810-1819, 2004.

[6] M. Anhalt and B Weidenfeller, "Dynamic losses in FeSi filled polymer bonded soft magnetic composites," Journal of Magnetism and Magnetic Materials, vol. 304, no. 2, pp. e549-e551, 2006.

[7] H. Skarrie, "Design of powder core inductors," University of Lund, Ph.D. dissertation 2001.

[8] A.H. Taghvaei, H. Shokrollahi, K. Janghorban, and H. Abiri, "Eddy current and total power loss separation in the iron-phosphate-polyepoxy soft magnetic composites," Materials and Design, vol. 30, no. 10, pp. 3989-3995, 2009.

[9] L.P. Lefebvre, S. Pelletier, and C. Gélinas, "Effect of electrical resistivity on core losses in soft magnetic iron powder materials," Journal of magnetism and magnetic materials, vol. 176, no. 2, pp. L93-L96, 1997.

[10] O. de la Barrière et al., "Loss separation in soft magnetic composites," Journal of Applied Physics, vol. 109, p. 07A317, 2011.

[11]J. Cros, P. Viarouge, and C Gelinas, "Design of PM brushless motors using iron-resin composites for automotive applications," in 33th IAS Annual Meeting, 1998, pp. 5-11.

[12]L.P. Lefebvre, S. Pelletier, B. Champagne, and C. Gélinas, "Effect of resin content and iron powder particle size on properties of dielectromagnetics," Advances in Powder Metallurgy and Particulate Materials, vol. 6, pp. 20.47-20.61, 1996.

[13]C. Gélinas, S. Pelletier, P. Lemieux, and L. Azzi, "Properties And Processing Of Improved SMC Materials," in Proceedings of the 2005 International Conference on Powder Metallurgy and Particulate Materials, 2005.

[14]L.P Lefebvre and C. Gélinas, "Effect of Material Insulation and Part Geometry on AC Magnetic Performances of P/M Soft Magnetic Composites," Advances in Powder Metallurgy and Particulate Materials, vol. 7, pp. 36-50, 2001.

[15]C. Cyr, P. Viarouge, J. Cros, and S. Clénet, "Resistivity measurement on soft magnetic composite materials," Przeglad Elektrotechniczny, vol. 83, no. 4, pp. 103-104, 2007.

[16]E. Barbisio, F. Fiorillo, and C. Ragusa, "Accurate measurement of magnetic power losses ans hysteresis loops under generic induction waveforms with minor loops," in Soft Magnetic Conference 16, 2003.

[17] M. De Wulf, L. Anestiev, L. Dupré, L. Froyen, and J. Melkebeek, "Magnetic properties and loss separation in iron powder soft magnetic composite materials," Journal of applied physics, vol. 91, pp. 7845-7847, 2002.

[18]H. Shokrollahi and K. Janghorban, "Soft magnetic composite materials (SMCs)," Journal of materials processing technology, vol. 187, no. 1, pp. 1-12, 2007.

[19]C. Appino et al., "Computation of eddy current losses in Soft Magnetic Composites," IEEE Transactions on Magnetics, vol. 48, no. 11, pp. 3470-3473, 2012. 
208 Fig. 1: Specific loss in $S M C_{2}$ samples (inorganic binder) as a function of frequency, for three 209 different thickness values $t_{1}, t_{2}$, and $t_{3}$ (sinusoidal polarization, $J_{\mathrm{p}}=1 \mathrm{~T}$ )

210 Fig. 2: Measured and calculated energy loss difference $\Delta W$ (sinusoidal polarization, $J_{\mathrm{p}}=1 \mathrm{~T}$ ) 211 between the $S M C_{1}-t_{3}$ and $S M C_{1}-t_{1}$ samples. The predicted $\Delta W$ behavior is obtained from Eq. (3) $212 \quad$ using the loss coefficient $Q^{(\text {loss })}=1$.

213 Fig. 3: As in Fig. 2 for the samples $S M C_{2}-t_{3}$ and $S M C_{2}-t_{1}$. The theoretical $\Delta W$ is obtained using $214 \quad Q^{(\text {loss })}=1.56$ in Eq. (3).

215 Fig. 4: Loss decomposition (sinusoidal polarization, $J_{\mathrm{p}}=1 \mathrm{~T}$ ) in the $S M C_{1}-t_{2}$ sample 216 Fig. 5: Loss decomposition (sinusoidal polarization, $J_{\mathrm{p}}=1 \mathrm{~T}$ ) in the $S M C_{2}-t_{2}$ sample 


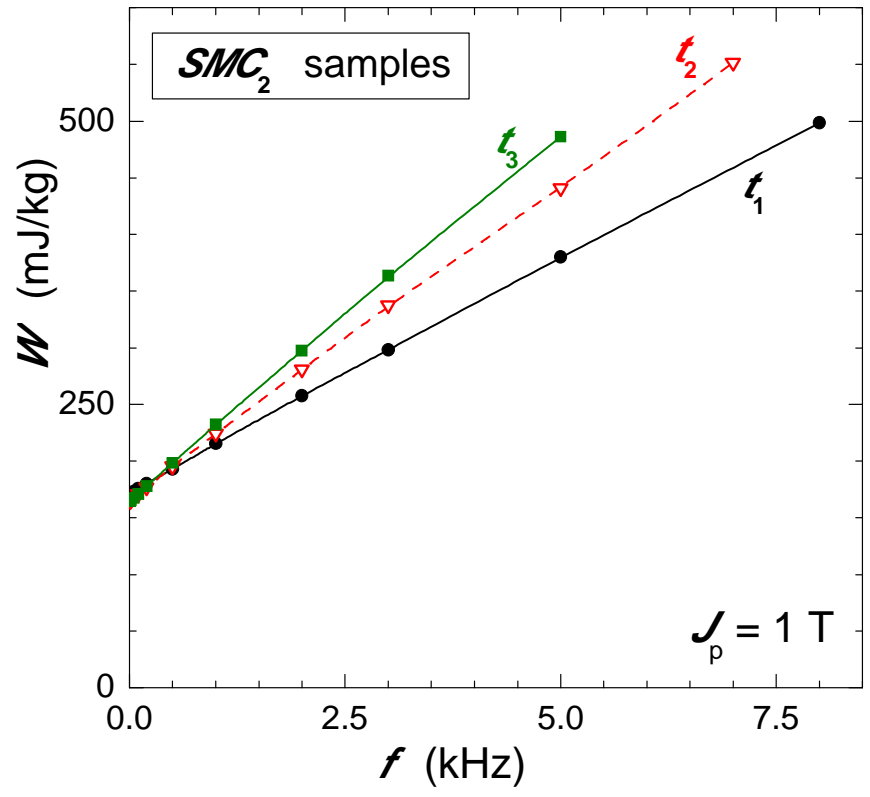

220

Fig. 1

221 


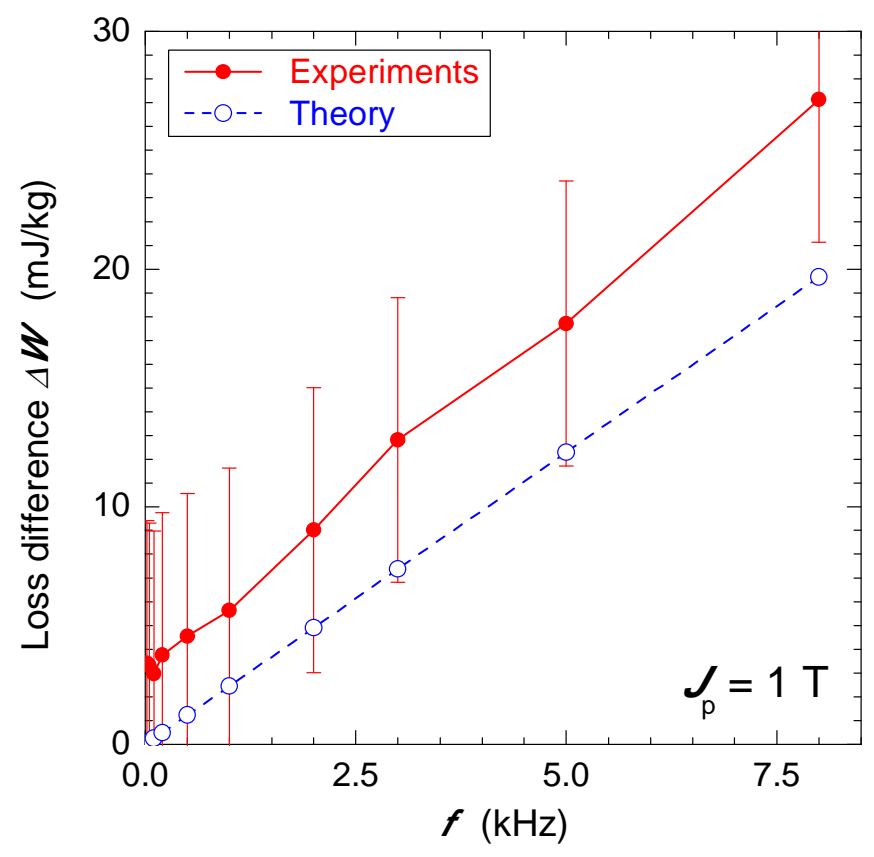

224

Fig. 2

225 


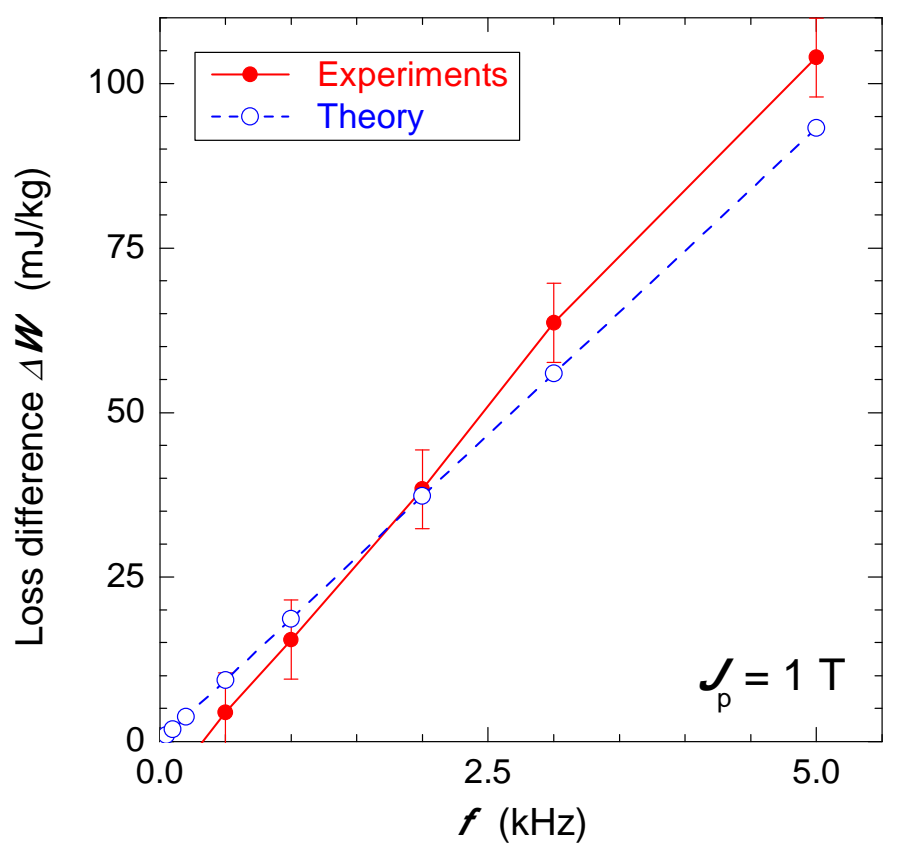

228

Fig. 3

229 


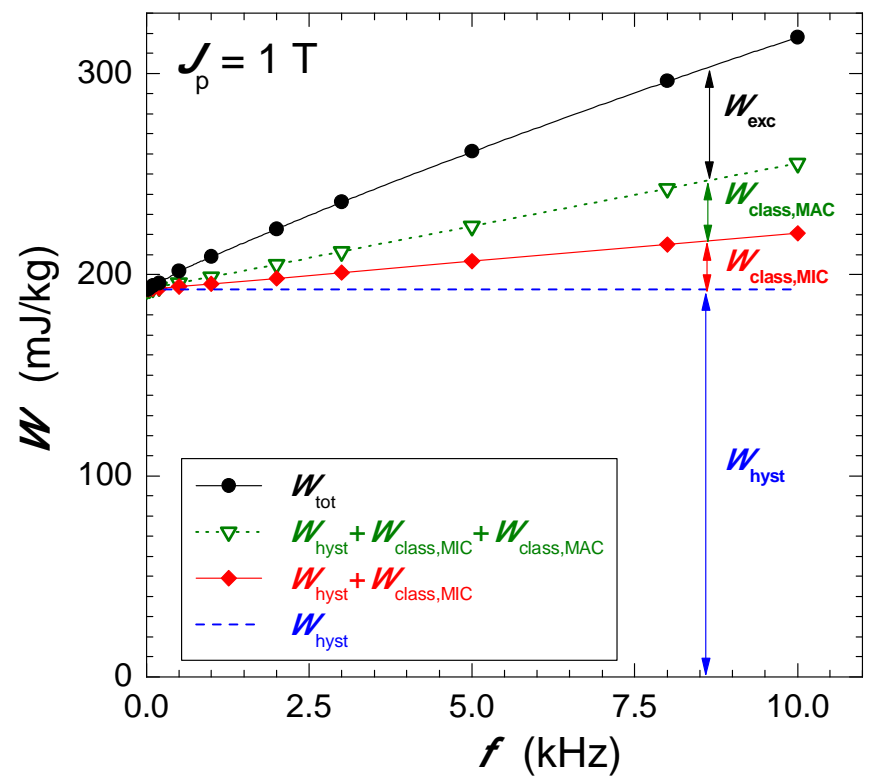

Fig. 4 


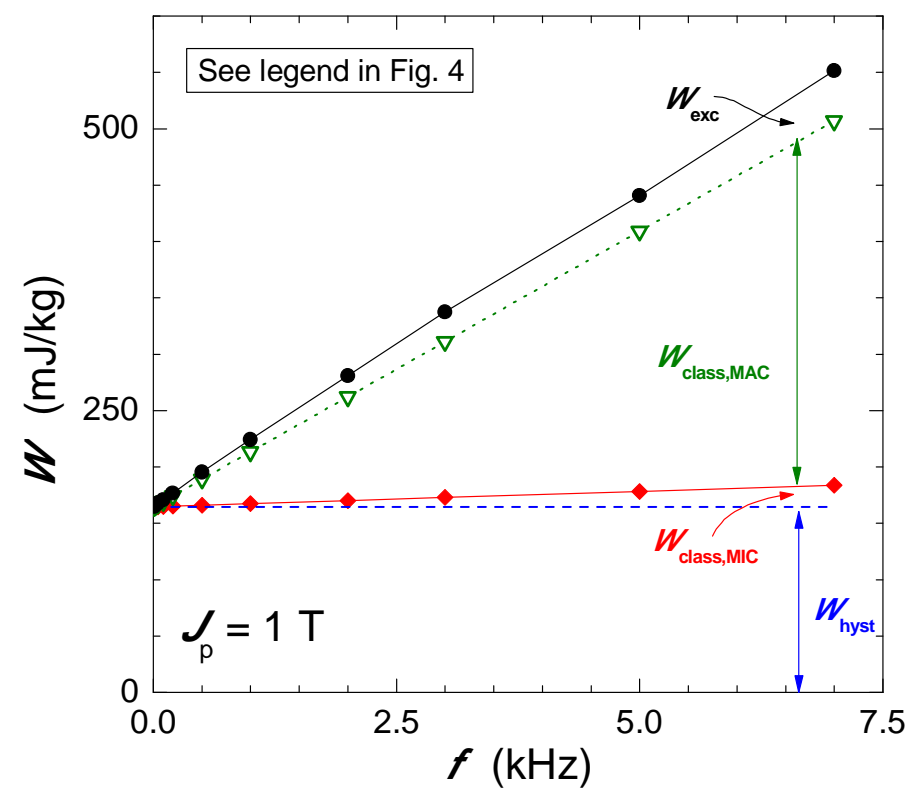

236

Fig. 5

237 


\section{Table captions}

239 Table 1: Obtained densities $\delta$ and resistivities $\rho$ for all materials $\left(S M C_{1}\right.$ and $\left.S M C_{2}\right)$ and thicknesses

$240 \quad\left(t_{1}, t_{2}\right.$ and $\left.t_{3}\right)$

241 
242

243

244

\begin{tabular}{|c|c|c|c|}
\hline \multirow{2}{*}{ Material } & \multicolumn{3}{|c|}{ Axial Thickness (mm) } \\
\cline { 2 - 4 } & $t_{1}=5$ & $t_{2}=9$ & $t_{3}=13$ \\
\hline \hline$S M C_{1}$ & $\delta=7110 \mathrm{~kg} / \mathrm{m}^{3}$ & $\begin{array}{c}\delta=7070 \\
\rho=911\end{array}$ & $\begin{array}{c}\delta=7010 \\
\rho=1170\end{array}$ \\
\hline \multirow{S}{*}{$S M C_{2}$} & $\delta=7590 \mu \Omega \cdot \mathrm{m}$ & $\rho 130$ & $\delta=7130$ \\
& $\rho=48$ & $\rho=43$ & $\begin{array}{c}\delta=7100 \\
\rho=45\end{array}$ \\
\hline
\end{tabular}

Table 1

245 